\title{
In vitro and in vivo Effects of Artesunate on Echinococcus granulosus Protoscoleces and Metacestodes
}

This article was published in the following Dove Press journal: Drug Design, Development and Therapy

\author{
Limei Wen ${ }^{1-3, *}$ \\ Guodong Lv $\mathrm{Lv}^{2,4, *}$ \\ Jun Zhaol,3,* \\ Shuai Lu ${ }^{1,3}$ \\ Yuehong Gong ${ }^{1,3}$ \\ Yafen $\mathrm{Li}^{2}$ \\ Haiya Zheng ${ }^{2}$ \\ Bei Chen 1,3 \\ Huijing Gao ${ }^{1,3}$ \\ Chunyan $\operatorname{Tian}^{2}$ \\ Jianhua Wang ${ }^{1,3}$
}

'Pharmaceutical Department, The First Affiliated Hospital of Xinjiang Medical University, Urumqi 830054, People's Republic of China; ${ }^{2}$ College of Pharmacy, Xinjiang Medical University, Urumqi 830054, People's Republic of China; ${ }^{3}$ State Key Laboratory of Pathogenesis, Prevention, Treatment of Central Asian High Incidence Diseases, Urumqi 830054, People's Republic of China; ${ }^{4}$ Clinical Medical Research Institute, The First Affiliated Hospital of Xinjiang Medical University, Urumqi 830054, People's Republic of China

*These authors contributed equally to this work
Background: In this study, we aim to investigate the efficiency of artesunate (AS) on Echinococcus granulosus protoscoleces and metacestodes.

Methods: For the in vitro assay, the eosin dye exclusion test and transmission electron microscope (TEM) were utilized to evaluate the effects of AS against protoscoleces (PSCs) from Echinococcus granulosus. In addition, mortality, ultrastructure change, reactive oxygen species (ROS) content and DNA damage were measured in order to explore the antiechinococcosis mechanism of AS. For the in vivo assay, CE-infected mice were divided into model group, albendazole (ABZ) group (200 mg/kg), low AS (AS-L) group (50 mg/kg), moderate AS (AS-M) group (100 mg/kg), and high AS (AS-H) group (200 mg/kg). Upon 6 weeks oral administration, wet weight of cysts and the ultrastructural changes of cystic wall were utilized to evaluate the effects of AS on metacestodes. In addition, the liver biochemical parameters, tumor necrosis factor- $\alpha$ (TNF- $\alpha$ ), glutathione/glutathione oxidized (GSH/GSSG) ratio in serum, and $\mathrm{H}_{2} \mathrm{O}_{2}$, total superoxide dismutase (T-SOD) in cyst fluid were detected. Results: Both in vivo and in vitro experiments showed that AS showed anti-parasitic effects on CE. The AS could elevate the ROS level in the PSCs, which then resulted in obvious DNA damages. AS could significantly improve the liver biochemical parameters in infected mice compared with the model group $(P<0.05)$. Compared with the model group, AS-M and AS-H decrease the TNF- $\alpha$ content $(P<0.05)$; AS-H group significantly decrease in the serum GSH/GSSG ratio $(P<0.05)$. The content of $\mathrm{H}_{2} \mathrm{O}_{2}$ in hydatid fluid treated by $\mathrm{AS}$ showed significant decrease compared with the model group $(P<0.01)$, while the T-SOD level showed significant elevation compared with model group $(P<0.01)$.

Conclusion: In this study, we confirmed that the effects of AS on Echinococcus granulosus protoscoleces and metacestodes may be related to the DNA damages induced by oxidative stress, which provided solid information for the research and development of drugs for cystic echinococcosis.

Keywords: cystic echinococcosis, artesunate, Echinococcus granulosus, reactive oxygen species, DNA damage

\section{Background}

Cystic echinococcosis (CE) is a zoonosis induced by the larval stage of Echinococcus granulosus sensu lato (E. granulosus), which is featured by longterm growth of hydatid cysts in mammalian intermediate hosts and human beings. ${ }^{1}$ The individuals may be infected accidentally via ingestion of eggs from feces of dogs infected with CE. The CE infected human mainly presents in the form of chronic hepatic disease. ${ }^{2}$ In endemic areas, the annual incidence of CE is in a range
Correspondence: Jianhua Wang Pharmaceutical Department, The First Affiliated Hospital of Xinjiang Medical University, No. 137 Liyushan South Road, Urumqi 830054, People's Republic of China Email jhw716@sohu.com 
of $<1$ to 200 per 100,000 inhabitants. ${ }^{3}$ Currently, it is estimated that the global burden is an average of 285,500 disability-adjusted life years for human CE.

According to the WHO-IWGE classification, four treatment and management options are suggested for CE, including surgery, percutaneous "PAIR" (percutaneous puncture, aspiration, injection, and reaspiration) technique, chemotherapy using benzimidazoles and "watch and wait" strategy. ${ }^{4}$ For the majority of cases, chemotherapy agents are necessary to improve the life quality of patients with a high possibility of recurrence, multiple-organ involvement as well as the cases of the advanced stages. ${ }^{5}$

Currently, benzimidazoles (eg, mebendazole and albendazole) are considered as the first option for chemotherapy. ${ }^{6}$ Albendazole (ABZ) was more effective than mebendazole because of its metabolite (ie, ABZ sulfoxide) that diffused easily from cyst membrane, which led to accumulation of $\mathrm{ABZ}$ in the cyst fluid. ${ }^{7}$ However, it really induces adverse effects such as nausea, vomiting, abdominal pain, diarrhea, dizziness, headache and gastro-intestinal disturbances. Also, it may cause abnormalities in liver functions, leukopenia and hematuria. Moreover, it may lead to hepatic injury after a long-term administration, together with decline of white blood cells. ${ }^{8}$ Limited and low efficient chemotherapeutic options are stumbling blocks to the treatment of CE. Hence, it is urgent to develop novel and effective chemotherapeutic agents.

Artemisinin (ART), an anti-malarial agent discovered in the 1970s, shows high efficacy and low toxicity for cancer patients. ${ }^{9}$ The active moiety of ART is a sesquiterpene lactone containing an endoperoxide bridge that can lead to generation of reactive oxygen species (ROS) responsible for mediating cytotoxic action of artemisinin derivatives in the parasites. ${ }^{10}$ According to the recent data, ROS was distributed in mitochondrial compartment in the parasites, which then resulted in impaired mitochondrial functions and DNA damages. ${ }^{11,12}$ The antiCE activity of ART derivatives involves direct DNA damages and subsequent parasite death. As the semisynthetic derivative of ART, artesunate (AS) has been reported to be involved in DNA damage and repair processes. ${ }^{13}$ In E. granulosus, we identified the ribosomal protein S9 from E. granulosus (EgRPS9) involved in DNA repair pathways. ${ }^{5}$ To date, many pharmacological actions and cellular targets of AS have been identified, ${ }^{14-16}$ but the anti-CE effects of AS are still ambiguous. In this study, we aim to investigate the pharmacodynamic roles of AS against $\mathrm{CE}$ and the potential mechanisms.

\section{Materials and Methods}

\section{PSCs Studies in vitro} Isolation of Parasites

E. granulosus sensu lato protoscoleces (PSCs) were aseptically isolated from liver hydatid cysts obtained from infected sheep scheduled for routine slaughter in an abattoir located in Urumqi (Xinjiang Autonomous Region, China), followed by pepsin-activation and in vitro culture according to our previous description. ${ }^{5}$ The pepsin solution at low $\mathrm{pH}$ was used for the incubation of larvae for 30 $\min$ at $37^{\circ} \mathrm{C}$. Then pepsin $(0.5 \mathrm{mg} / \mathrm{mL})$ was added to a $0.85 \%(\mathrm{w} / \mathrm{v})$ sodium chloride solution to prepare the pepsin solution (pH 2.0). Subsequently, RPMI1640 medium (Gibco/BRL, Rockville, Maryland) was utilized for the culture of PSCs, supplementing with $100 \mathrm{U} / \mathrm{mL}$ penicillin, $10 \%$ fetal bovine serum (FBS; Gibco/BRL), and 100 $\mathrm{lg} / \mathrm{mL}$ streptomycin (Gibco/BRL) at $37^{\circ} \mathrm{C}$ in a humidified $5 \% \mathrm{CO}_{2}$ incubator.

\section{Evaluation of Pharmaceutical Efficiency of AS on PSCs}

After culturing for $24 \mathrm{hrs}$, the PSCs were collected and washed with PBS thrice. PSCs with a viability of $\geq 95 \%$ by eosin dye exclusion test were used for the subsequent analysis. ${ }^{17}$ About 250 PSCs were seeded onto each well of the 96 -well plates by dividing into the following groups: control group, plates supplemented with cultivation medium; DMSO group, treated with dimethyl sulfoxide (DMSO, $1 \%$, v/v); $\mathrm{H}_{2} \mathrm{O}_{2}$ group, treated with $50 \mu \mathrm{M} \mathrm{H}_{2} \mathrm{O}_{2}$ (as a positive control for ROS); ABZ group $(25 \mu \mathrm{M}$, as positive drugs); and AS groups. According to the previous description, ${ }^{10}$ the $\mathrm{LC}_{50}$ of AS for the PSCs was $130 \mu \mathrm{M}$, while that for the low dose AS (AS-L), middle dose AS (AS$\mathrm{M})$, and high dose AS (AS-H) was $1 / 2 \mathrm{LC}_{50}(65 \mu \mathrm{M}), 1$ $\mathrm{LC}_{50}(130 \mu \mathrm{M})$, and $2.5 \mathrm{LC}_{50}(325 \mu \mathrm{M})$, respectively.

After treating for 4 days, PSCs were collected to evaluate the mortality via the eosin dye exclusion test, followed by monitoring the ultramicroscopic change by transmission electron microscopy (TEM, JEM-100CXII, Alignment, Japan) as previously described. ${ }^{18,19}$

\section{Effect of AS on PSCs Mortality in vitro}

PSCs were divided into the following groups: DMSO group; $\mathrm{H}_{2} \mathrm{O}_{2}$ group; $\mathrm{ABZ}$ group $(25 \mu \mathrm{M})$; and $\mathrm{AS}-\mathrm{H}$ group $(325 \mu \mathrm{M}) ; \mathrm{H}_{2} \mathrm{O}_{2}$ plus Mannitol group, treated with 
$50 \mu \mathrm{M} \mathrm{H}_{2} \mathrm{O}_{2}$ and $100 \mu \mathrm{M}$ mannitol (scavenger of ROS); AS-H plus mannitol group, treated with $325 \mu \mathrm{M}$ AS and $100 \mu \mathrm{M}$ mannitol. After treating for 4 days, PSCs were collected to evaluate the mortality and observe the ultramicroscopic changes.

\section{Detection of ROS Content in PSCs of E. granulosus}

Generation of ROS in PSCs was determined as previously described..$^{20}$ Briefly, the dihydrorhodamine 123 (DHR123, D1054, Sigma-Aldrich) was added to the 96-well plates covered with PSCs to a final concentration of $10 \mu \mathrm{M}$. Then the mixture was incubated at $37^{\circ} \mathrm{C}$ for $30 \mathrm{~min}$. Upon removal of the supernatant, the mixture was washed using PBS once, followed by incubating with agents at $37^{\circ} \mathrm{C}$. The wavelength was set at $530 \mathrm{~nm}(\lambda \mathrm{ex}=488 \mathrm{~nm})$. A multifunction reader (Thermo Fisher) was utilized for the observation for $5.5 \mathrm{hrs}$ every $30 \mathrm{~min}$.

\section{Detection of DNA Damage by Comet Assay}

Optimized comet assay for E. granulosus was carried out according to method for Plasmodium falciparum described by Gopalakrishnan et $\mathrm{al}^{21}$ with some minor modifications. The PSCs were treated for $24 \mathrm{hr}$, the culture medium was abandoned and washed using PBS. Normal-melting point agarose (NMA) was transferred onto the slide, and incubated overnight at $65^{\circ} \mathrm{C}$ to solidify the agarose. Diluted PSCs (100) were mixed with $100 \mu \mathrm{L}$ low-melting point agarose (LMA) added onto the slides which were then immediately covered with coverslips. After agarose solidification at $4^{\circ} \mathrm{C}$ for $5 \mathrm{~min}$, the coverslips were removed and the slides were immersed for $4 \mathrm{hrs}$ at $4^{\circ} \mathrm{C}$ in freshly lysis solution. The slides were equilibrated in alkaline solution for $20 \mathrm{~min}$. Electrophoresis was carried out for $20 \mathrm{~min}$ at $25 \mathrm{~V}$ and $300 \mathrm{~mA}$. Afterwards, slides were neutralized by washing with Tris- $\mathrm{HCl}$ buffer for $15 \mathrm{~min}$, and were stained with $60 \mu \mathrm{L}$ propidium iodide (PI) solution in dark. Finally, the images were observed using a fluorescent microscope (Olympus IX73) connected through a CCD-IRIS Color Video Camera (Hitachi Denshi, Japan), under a magnification of $200 \times$. The images for PSCs were acquired immediately after opening the microscope shutter to the computer monitor, employing the CASP Program.

\section{Animal Studies}

Female Kunming mice ( 6 weeks old) provided by the Animal Center, Xinjiang Medical University, were subject to adaptive feeding for one week before experiments. All protocols involving animals were approved by the Animal Welfare and Committee of First Affiliated Hospital of Xinjiang Medical University (IACUC-20150225-70). Mice were infected by intraperitoneal injection 25 small vesicles with a diameter of $250-300 \mu \mathrm{m}$ cultured from PSCs of E. granulosus in vitro as previously described. ${ }^{22,23}$ Six months later, selection of infected mice was performed under ultrasonographic examinations.

\section{Experimental Grouping and Administration}

The infected mice were randomly divided into: (i) model group ( $\mathrm{n}=10)$ : treated with Tween-80/0.4\% CMC-Na; (ii) ABZ positive control group $(n=10)$ : treated with $A B Z$ (200 mg/kg); and (iii) AS groups $(\mathrm{n}=30)$ : treated with $50 \mathrm{mg} / \mathrm{kg} \quad\left(1 / 20 \mathrm{LD}_{50}\right), \quad 100 \mathrm{mg} / \mathrm{kg} \quad\left(1 / 10 \mathrm{LD}_{50}\right)$ and $200 \mathrm{mg} / \mathrm{kg}\left(1 / 5 \mathrm{LD}_{50}\right)$ of AS. Uninfected mice treated with an equal volume of $0.4 \% \mathrm{CMC}-\mathrm{Na}$ served as control group $(n=10)$. The solution was given by intragastric administration lasting for 6 weeks (once per day).

Six weeks later, the blood samples were obtained by cardiac puncture after anesthesia with pentobarbital sodium via intraperitoneal administration. ${ }^{24}$ Serum samples were collected to measure the liver biochemical parameters to evaluation liver injury. Serum tumor necrosis factor- $\alpha(\mathrm{TNF}-\alpha)$ and glutathione/glutathione oxidized (GSH/GSSG) ratio was determined to evaluate the oxidative stress status of the whole body.

The mice were sacrificed by cervical dislocation immediately after blood collection, and the cysts were isolated and subject to measurement of the wet weight of cysts. The cyst fluid was transferred by a syringe for $\mathrm{H}_{2} \mathrm{O}_{2}$ and total superoxide dismutase (T-SOD) measurement to evaluate the oxidative stress status in the cysts. The cystic wall was observed under the TEM to monitor the ultrastructural changes.

\section{TEM}

For cystic wall ultrastructural changes after drug treatments, cysts were processed for TEM analysis as described previously. ${ }^{25}$ Micrographs were taken on a JEM-100CX II TEM.

\section{Liver Biochemical Parameters Measurement}

Liver biochemical parameters mainly included the content of total bilirubin (TBIL) and direct bilirubin (DBIL) levels, alanine aminotransferase (ALT), and aspartate aminotransferase (AST). TBIL and DBIL levels were evaluated using commercial kits (Cat: C019-1; Cat: C019-2; Jiancheng Biotech, Nanjing, China), according the manufacturer's instructions. 
Both the detection wavelength was set at $450 \mathrm{~nm}$. The ALT and AST levels were measured using commercial kits (Cat: C010-2; Cat: C009-2; Jiancheng Biotech, Nanjing, China). The absorbance was measured under a wavelength of 510 $\mathrm{nm}$. All the tests were performed at least in triplicate.

\section{Determination of TNF- $\alpha$}

The mouse serum TNF- $\alpha$ level was determined by the ELISA Kit (Cat: CSB-E04741m, Cusabio Biotech, Wuhan China). The absorbance was measured using a multi-detection microplate reader (Thermo Fisher Scientific Inc.) at a wavelength of $450 \mathrm{~nm}$.

\section{Oxidative Stress Status Evaluation}

Determination of GSH/GSSG Ratio

The mouse serum GSH/GSSG ratio was determined by GSH/GSSG Ratio Detection Assay Kit (Cat: A061-1, Jiancheng Biotech, Nanjing, China) according to the manufacturer's instructions. The plate was incubated at room temperature for 10-60 min in dark. The fluorescence was monitored at $\mathrm{Ex} / \mathrm{Em}$ of $490 / 520 \mathrm{~nm}$ with a microplate reader.

\section{Determination of $\mathrm{H}_{2} \mathrm{O}_{2}$ and T-SOD}

The cyst fluid centrifugation at $3000 \mathrm{rpm} /$ minute for 15 min. Supernatant was collected, followed by $\mathrm{H}_{2} \mathrm{O}_{2}$ and T-SOD content detection using the Hydrogen Peroxide assay kit (A064; Nanjing Jiancheng Biotech, Nanjing, China) and the T-SOD assay kit (A001-1, Nanjing Jiancheng Biotech, Nanjing, China) according to the manufacturer's instructions. The absorbance was monitored at a wavelength of $405 \mathrm{~nm}$ and $420 \mathrm{~nm}$, respectively.

\section{Statistical Analysis}

Data analysis was performed using SPSS 18.0, and measurement data were presented as mean \pm standard error, and numeration data were presented as percentages. All data were obtained from the experiments performed at least in triplicate. The comparison was analyzed with oneway ANOVA and $\chi^{2}$ test, respectively. $P<0.05$ was considered to be statistically significant.

\section{Results}

\section{Pharmacodynamic Features of AS on PSCs in vitro}

Eosin dye exclusion test was used to measure the mortality rate of PSCs after treatment (Figure 1A). Compared with the DMSO group, the protoscolicidal activity in AS group and ABZ group were all satisfactory. The changes of protoscolicidal activity in the AS group presented a dose-dependent manner. Compared with the ABZ group, significant increase was noticed in the mortality was observed in AS groups $(P<$ $0.05)$. On day 4 , TEM was performed to monitor the ultrastructural changes of PSCs. In the control and DMSO groups, the laminar layer (11) were intact, together with the microtrichies (mt) (Figure 1B). There were no aberrant changes in the parenchyma cells, and the structures were clear. In the ABZ group, the mt was no longer available, the membrane structure was turbid. The cell nucleus was no longer available and lipid droplets were observed. In the low AS group (AS-L group), few microvillus were observed, and the nucleolus was disperse. In the AS-M group, there were some heterochromatins that were hallmarks for the DNA damages. In the

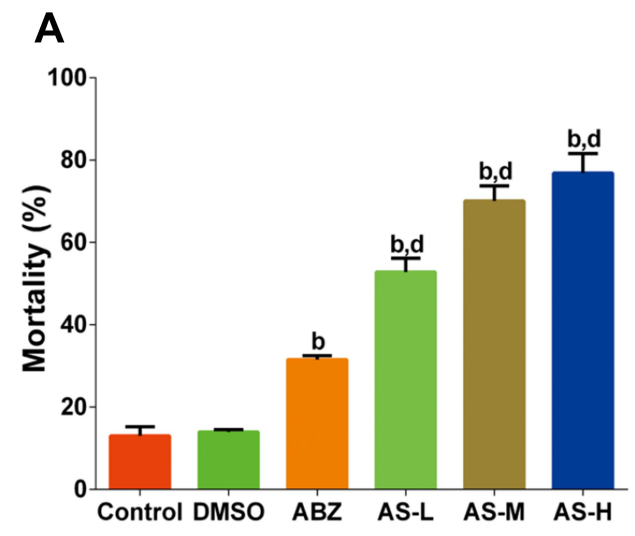

B
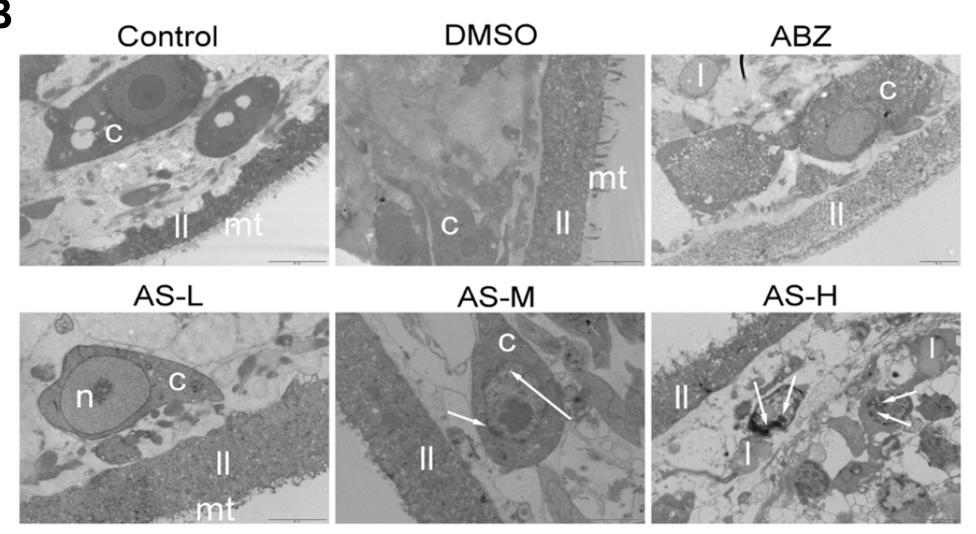

Figure I Effects of AS on mortality and ultrastructural changes of $E$. granulosus. (A) Mortality of PSCs on day 4 in each group using eosin dye exclusion test. (B) Ultrastructural changes under transmission electron microscope in $(12,000 \times)$, DMSO group $(10,000 \times)$, ABZ group $(8000 \times)$, AS-L group (I2,000 ×), AS-M group $(12,000$ $\times)$, and AS-H group $(10,000 \times) .{ }^{b} P<0.01$ versus DMSO group; ${ }^{d} P<0.01$ versus $A B Z$ group.

Abbreviations: gl, germinal layer; c, parenchyma cell; mt, microtrichies; $n$, nucleolus; I, lipid droplet; heterochromatins highlighted by arrows. 
AS-H group, there were massive heterochromatins. This indicated that AS could interrupt the karyotin in the PSCs.

\section{Effects of AS on the DNA Oxidative Damages in PSCs in vitro}

The mortality in the DMSO group and ABZ group was $13.9 \pm$ $0.60 \%$ and $31.55 \pm 0.95 \%$, respectively (Figure 2A).
Compared with the $\mathrm{H}_{2} \mathrm{O}_{2}$ group, significant decrease was observed in the $\mathrm{H}_{2} \mathrm{O}_{2}+$ mannitol group $(P<0.01)$. Compared with the AS-H group, the mortality in the AS-H plus mannitol group showed significant decrease $(P<0.01)$. For the ultrastructure, mannitol could significantly attenuate the injuries of mt, 11 and nucleolus by $\mathrm{H}_{2} \mathrm{O}_{2}$ and $\mathrm{AS}$ (Figure 2B).

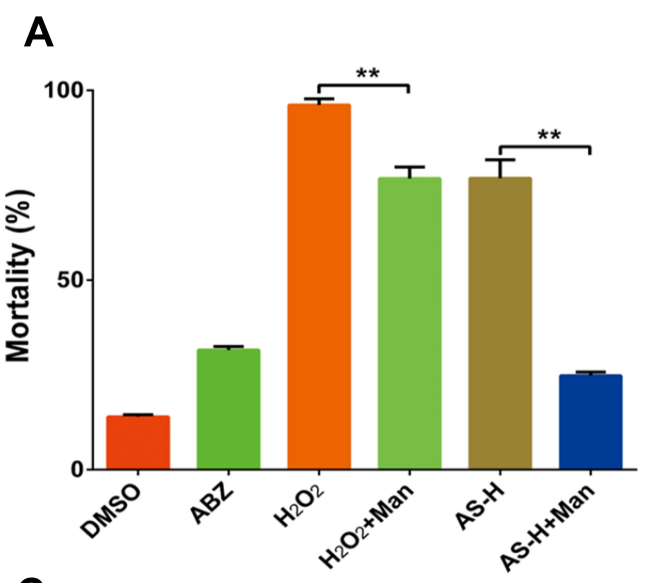

C

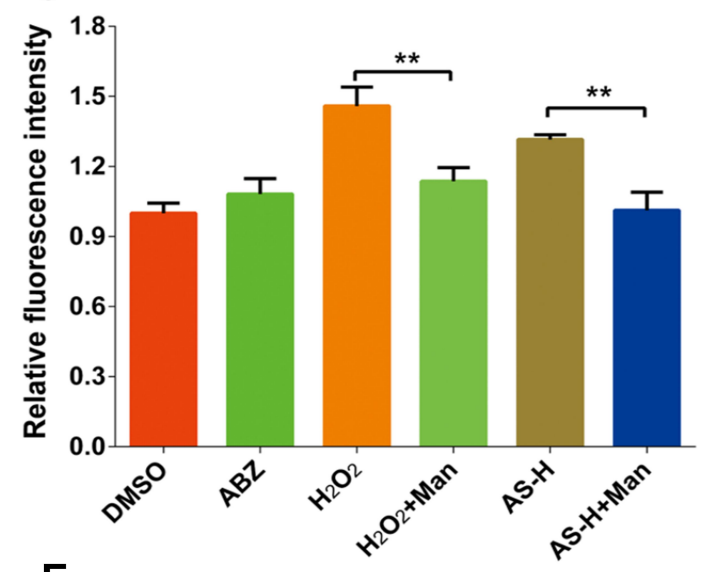

E
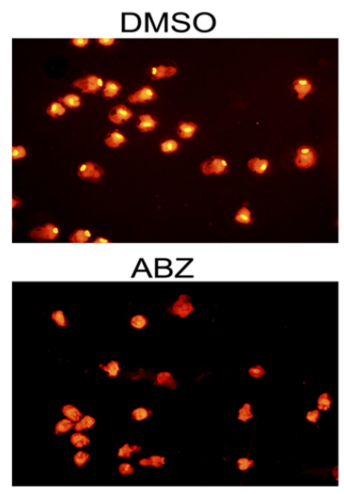

$\mathrm{H}_{2} \mathrm{O}_{2}$
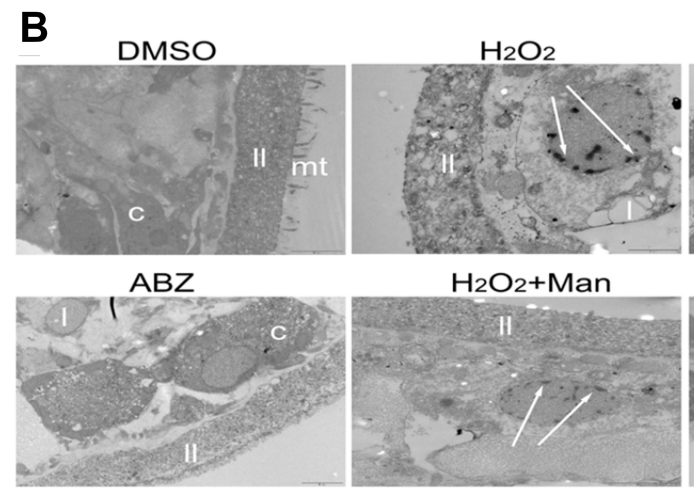

$\mathrm{H}_{2} \mathrm{O}_{2}+\mathrm{Man}$
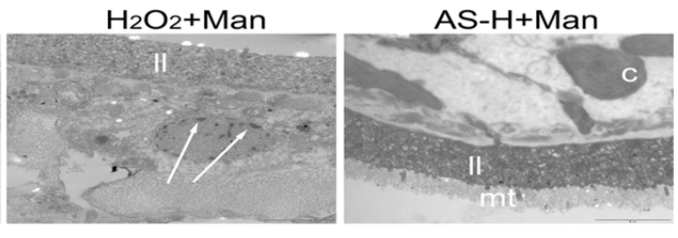

D
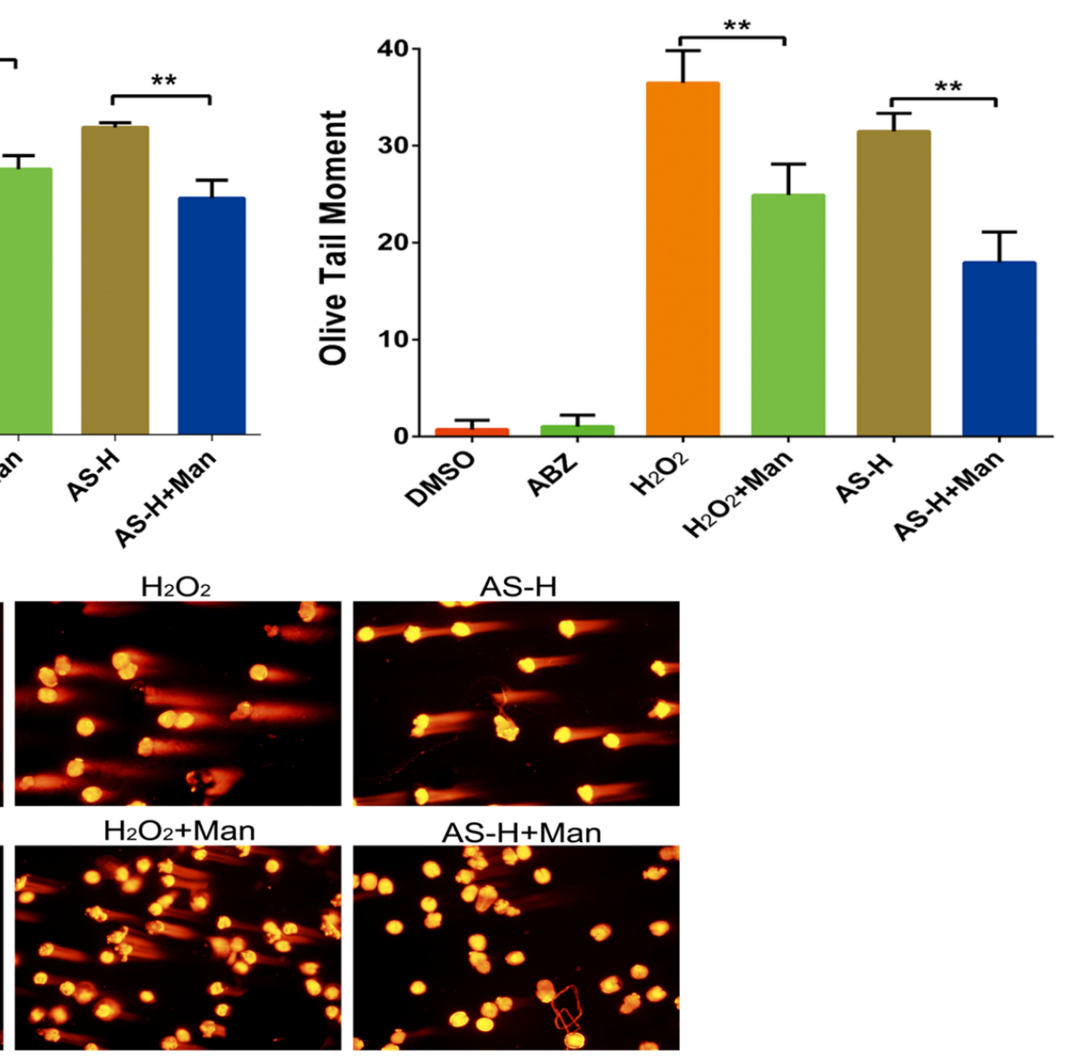

Figure 2 DNA oxidative damage effects of AS on E. granulosus. PSCs were treated 4 days and then collected to detect the mortality (A), and observe ultrastructural changes under transmission electron microscope in DMSO group $(10,000 \times), \mathrm{H}_{2} \mathrm{O}_{2}$ group $(20,000 \times)$, AS- $\mathrm{H}$ group $(10,000 \times), A B Z$ group $(8000 \times), \mathrm{H}_{2} \mathrm{O}_{2}+$ mannitol group $(20,000 \times)$, and AS- $\mathrm{H}+$ mannitol group $(12,000 \times)(B)$. ROS content in PSCs (C) tabbed by dihydrorhodamine I23, and DNA damage (D and E) established by Comet assay. **P < 0.0 I. Abbreviations: gl, germinal layer; c, parenchyma cell; mt, microtrichies; n, nucleolus; I, lipid droplet; heterochromatins highlighted by arrows. 
In Figure 2C, we determined the content of ROS within $30 \mathrm{~min}$ after drugs treatment, which indicated that AS could trigger the elevation of ROS in PSCs. Significant decrease was observed in the ROS content in the AS combined with mannitol group $(P<0.01)$. Comet assay revealed that AS could induce obvious DNA damages in PSCs (Figure 2D). After AS combined with the mannitol, obvious decrease was observed in the DNA injury damages $(P<0.01)$. These implied that mannitol could inhibit the anti-parasitic effects of AS on PSCs, which indicated that the anti-parasitic effects of AS may be related to the ROS-associated DNA injuries (Figure 2E).

\section{Pharmacodynamic Features of AS Against CE in vivo}

Cyst weight results are shown in Figure 3A. The cyst weight of model group was $6.76 \pm 2.51 \mathrm{~g}$, while that of the ABZ group was $1.76 \pm 0.97 \mathrm{~g}$. The cyst weights in the AS-L, AS-M and AS-H groups were $4.3 \pm 1.26 \mathrm{~g}, 3.29 \pm$ $1.4 \mathrm{~g}$, and $2.99 \pm 2.19$, respectively. Significant improvements were obtained after treating with ABZ, AS-L, AS-M and AS-H $(P<0.01)$. In addition, the ultrastructure of cystic wall was observed under the TEM (Figure 3B). The $\mathrm{mt}$ in the model group was intact. The nucleus was large and round. The laminar layer structure was even. The $\mathrm{mt}$ in the ABZ group was no longer available, but there were some lipid droplets. The structure of the AS-H group was not regular, and the mt was no longer available. Especially, massive lipid droplets were observed. The injury of the cystic wall tissues in the AS-H group was more severe than that of the $\mathrm{ABZ}$ group.

\section{Effects of AS on the Liver Biochemical Parameters}

Liver biochemical parameters indicated that, compared with the model group, the TBIL and AST levels showed significant decrease in the AS groups $(P<0.05$, Figure 4A). Compared with the model group, significant decrease was noticed in the DBIL and ALT in the AS-M and AS-H groups $(P<0.05)$. Compared with the ABZ group, significant decline was observed in the TBIL in the AS-M and AS-H groups $(P<0.01)$. These indicated that the liver protective effects of AS against mice with $\mathrm{CE}$ were similar with those of ABZ.

\section{Effects of AS on the TNF- $\alpha$ Level}

The serum TNF- $\alpha$ showed elevation in CE-infected mice (Figure 4B). Compared with the model group, significant decreases were noticed in the TNF- $\alpha$ in the ABZ, AS-M and AS-H groups $(P<0.05)$. The TNF- $\alpha$ showed significant decline in the AS-H group compared to that of the ABZ group $(P<0.01)$.

\section{Effects of AS on the Oxidative Damage on $C E$ in vivo}

Serum GSH/GSSG assay revealed that the oxidative stress showed a tendency of decrease in the infected mice treated with AS (Figure 4C). Compared with the model group,
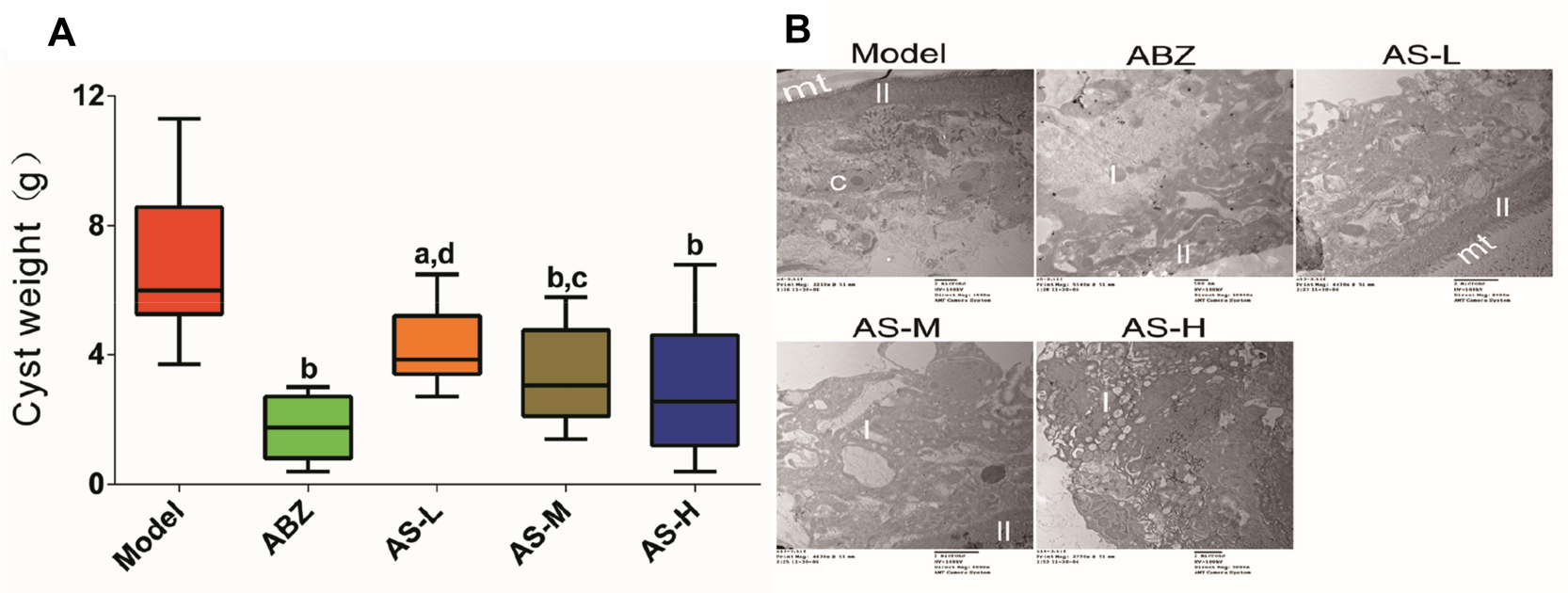

Figure 3 Experimental chemotherapy with E. granulosus-infected mice. (A) Cyst weight from mice at week 6. (B) Cyst wall observed under transmission electron microscope in model Group $(4000 \times)$, ABZ group $(5000 \times)$, AS-L $(5000 \times)$, AS-M $(5000 \times)$, and AS-H $(8000 \times)$. ${ }^{a} P<0.05$ versus model group; ${ }^{b} P<0.01$ versus model group; ${ }^{c} P<0.05$ versus $A B Z$ group; ${ }^{d} P<0.01$ versus $A B Z$ group.

Abbreviations: gl, germinal layer; c, parenchyma cell; $\mathrm{mt}$, microtrichies; $\mathrm{n}$, nucleolus; I, lipid droplet. 
A
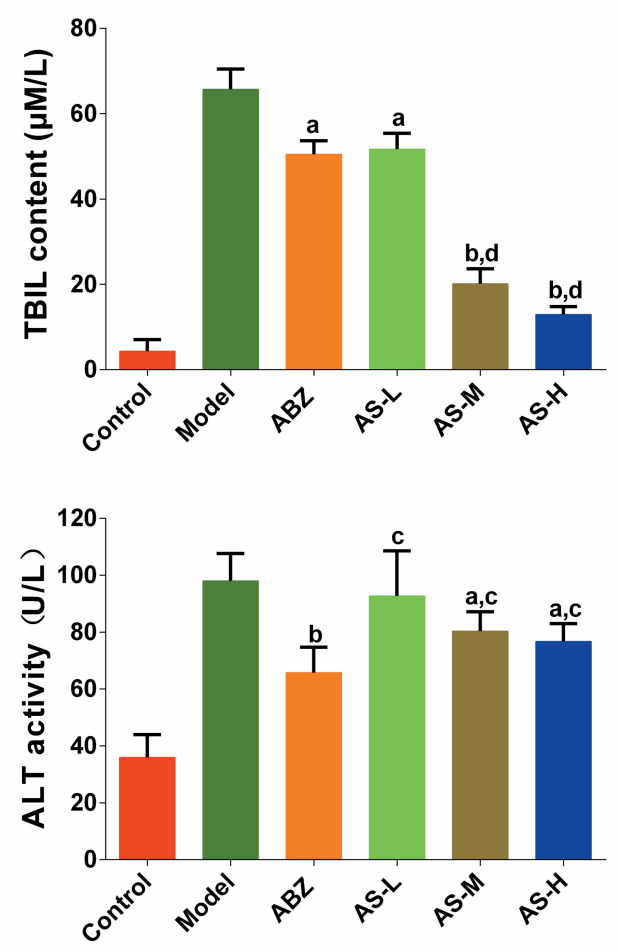

B

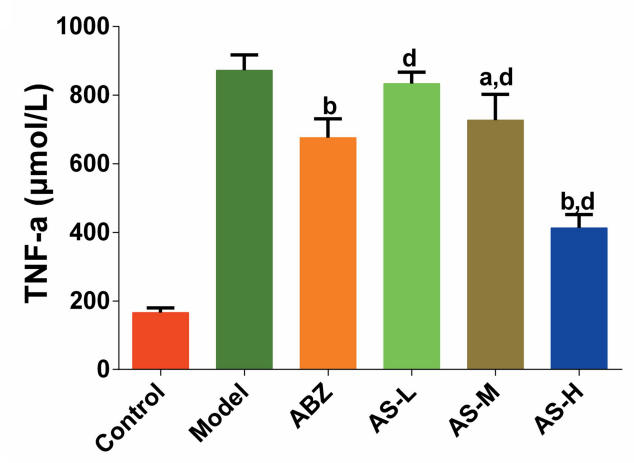

D

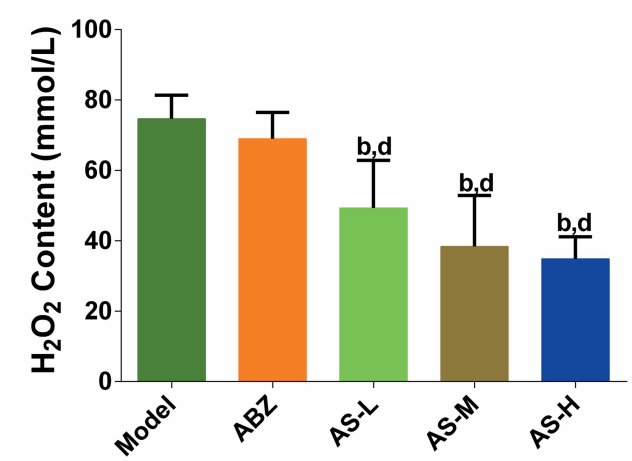

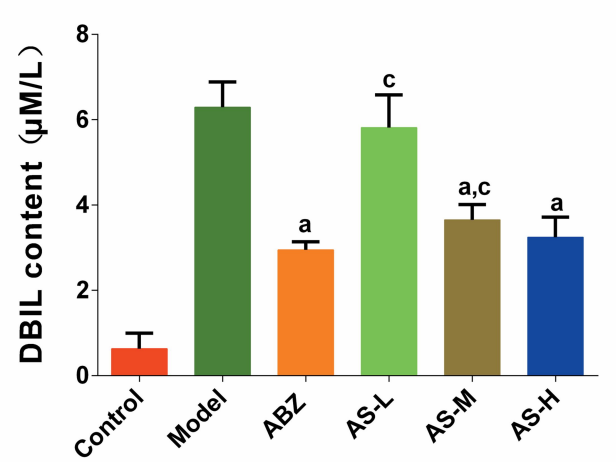

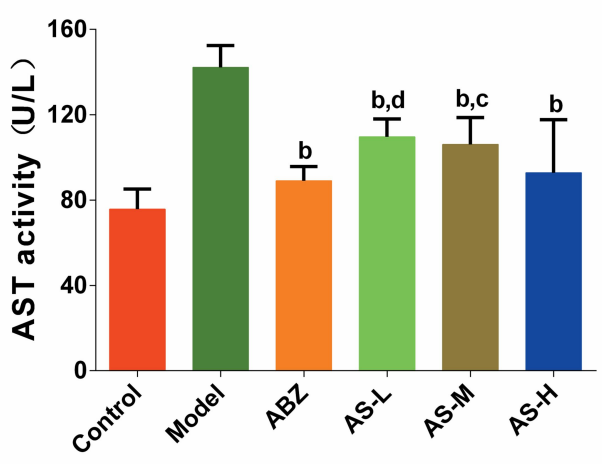

C

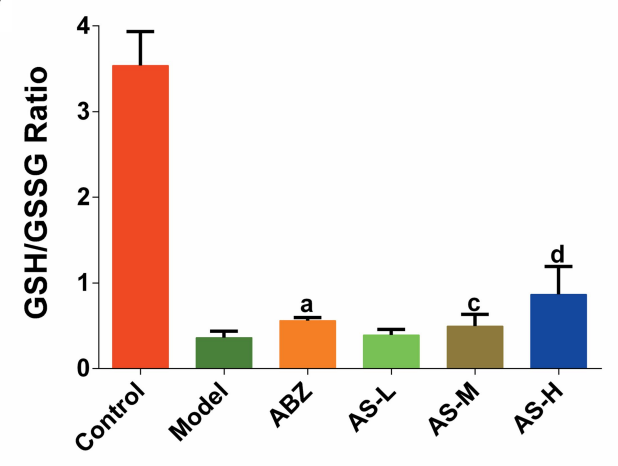

E

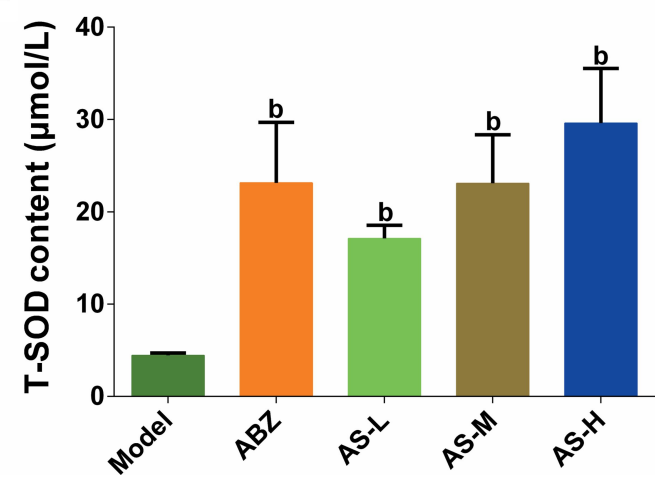

Figure 4 Molecular indices in serum or cyst fluid detection. (A) Liver biochemical parameters. (B) Effects of AS on TNF- $\alpha$ content. (C) Effects of AS on GSH/GSSH ratio. (D) Effects of $\mathrm{AS}$ on $\mathrm{H}_{2} \mathrm{O}_{2}$ content. (E) Effects of AS on T-SOD content. ${ }^{\text {a }} P<0.05$ versus model group; ${ }^{b} P<0.01$ versus model group; ${ }^{\mathrm{c}} P<0.05$ versus $A B Z$ group; ${ }^{\text {d }} P<0.0$ I versus $A B Z$ group. 
there were significant decreases in the serum GSH/GSSG ratio in the AS-H group $(P<0.05)$.

AS could decrease the content of $\mathrm{H}_{2} \mathrm{O}_{2}$, compared with the model group $(P<0.01)$. Compared with $\mathrm{ABZ}$ group, AS could significantly decrease the content of $\mathrm{H}_{2} \mathrm{O}_{2}(P<$ 0.01 , Figure 4D). In the CE-infected mice, the T-SOD content in the cystic fluid showed increase to some extent (Figure 4E). Compared with the model group, T-SOD showed significant increase in the ABZ group and AS groups $(P<0.01)$. There were no statistical differences in the T-SOD between the AS group and ABZ group.

\section{Discussion}

To our knowledge, we evaluated the effects of AS in vitro by eosin staining, and in vivo by wet weight of cysts from mice. We also investigated the ROS generation by DHR 123 method, DNA damage by comet assay, respectively. In addition, mannitol was used as ROS scavenger ${ }^{10}$ to identify the potential anti-echinococcosis role of ART. Our data showed that AS showed anti-echinococcosis effects in vitro and in vivo. Meanwhile, ROS level showed elevation after AS treatment, which presented in a dose-dependent manner. Similarly, comet assay showed that $\mathrm{H}_{2} \mathrm{O}_{2}$ and AS could induce DNA damages in PSCs. The DNA damage showed attenuation in the combination of AS combined with ROS scavenger, and the mortality showed significant decline. These data implied that the anti-parasitic effects of AS on PSCs may be related to the DNA damages induced by ROS generation. This paves the way for the development of new drugs targeting CE based on AS.

Comet assay, initially reported by Ostling et al in 1984, was utilized in the determination of DNA strand damages. ${ }^{26}$ However, it is not used in the evaluation of DNA damages in E. granulosus. In this study, it was used for the evaluation of PSCs for the first time after multiple optimizations, including the gel procedures, time for the lysis, staining dye selection, as well as the rinse frequency and time. This paves the way for the comet assay for the DNA damage evaluation in the PSCs.

Wet weight of cysts and ultrastructure evaluation under TEM were used as the indices for evaluating the in vivo potency. Our data showed that AS could inhibit the lesion growth of the $\mathrm{CE}$ in mice. The efficiency of AS in vitro was far more than ABZ, while the efficiency of low dose AS was comparable to that of ABZ. This may be related to the fact that $\mathrm{ABZ}$ may metabolize into the $\mathrm{ABZ}$ sulfoxide. Spicher et $\mathrm{al}^{17}$ reported that AS showed obvious effects against PSCs from E. granulosus. Whereas, it was not effective for the E. multilocularis-infected mice. Unlike the previous study, we utilized the E. granulosus-infected mice, which indicated AS-H group inhibited the growth of CE. It showed similar effects with $\mathrm{ABZ}$, which was in line with our previous study. ${ }^{27}$

In this study, cystic fluid was preferred for the determination of indices associated with oxidative stress, in order to investigate the potency of AS. Preliminary tests showed that only T-SOD and $\mathrm{H}_{2} \mathrm{O}_{2}$ were measurable, and only the $\mathrm{H}_{2} \mathrm{O}_{2}$ showed decrease in the fluid in the AS groups compared with the $\mathrm{ABZ}$ group. Whereas, the T-SOD activity in AS groups was significantly higher than that of ABZ group, indicating that AS could promote the anti-oxidant capacity of PSCs. On this basis, we speculated that AS induced remission of CE may be related to the oxidative stress improvement in the cysts.

Indeed, there are some limitations in this study. For example, only T-SOD and $\mathrm{H}_{2} \mathrm{O}_{2}$ could be detected in the cystic fluid. As there were no PSCs in the cyst in infected mice, we can not confirm the definite presence of DNA damages in vivo. As is known to all, long-term administration of $\mathrm{ABZ}$ for at least 6 months may lead to generation of hepatic injury caused by adverse events. In this study, after a 6-month administration of ABZ, there was severe hepatic injury in the model group. Animals treated using AS could significantly improve the liver function in the animals administrated using ABZ. This may be related to the fact that both $\mathrm{ABZ}$ and $\mathrm{AS}$ were effective for treating $\mathrm{CE}$, which was beneficial compared to the hepatic injury induced by these agents. In our subsequent study, we will focus on the DNA damages of PSCs in the mice infected with CE, together with the determination of oxidative damage indices.

In summary, AS was superior to the $\mathrm{ABZ}$ in treating PSCs infection in vitro. AS could significantly increase the oxidative stress and DNA damages. On the contrary, The anti-CE effect of AS was not superior to $\mathrm{ABZ}$ in vivo. In addition, the combination of AS and mannitol could significantly attenuate the DNA injuries, which implied that the potency of AS may be related to the regulation of DNA damages induced by oxidative stress in PSCs. This study provides solid information for the research and development of drugs for CE.

\section{Abbreviations}

ABZ, albendazole; ART, Artemisinin; AS, artesunate; ALT, alanine aminotransferase; AST, and aspartate aminotransferase; CE, cystic echinococcosis; DBIL, direct bilirubin; GSH/GSSG, glutathione/glutathione oxidized; $\mathrm{H}_{2} \mathrm{O}_{2}$, 
hydrogen peroxide; LMA, low-melting point agarose; NMA, normal-melting point agarose; PI, propidium iodide; PSCs, protoscoleces; ROS, reactive oxygen species; RPS9, ribosomal protein S9; TBIL, total bilirubin; TEM, transmission electron microscope; TNF- $\alpha$, tumor necrosis factor- $\alpha$; T-SOD, total superoxide dismutase.

\section{Data Sharing Statement}

The datasets used and/or analysed during the current study are available from the corresponding author on reasonable request.

\section{Ethics Approval and Consent to Participate}

All applicable international, national, and/or institutional guidelines for the care and use of animals were followed.

\section{Consent for Publication}

Informed consent was obtained from all individual participants included in the study.

\section{Funding}

This work was supported by the National Natural Science Foundation of China (Nos. 81560607 and 81860666), State Key Laboratory of Pathogenesis, Prevention, Treatment of Central Asian High Incidence Diseases Fund (No. SKL-HIDCA-2017-Y7), and Xinjiang Pharmaceutical Association Fund (No. YXH201704).

\section{Disclosure}

The authors declare that they have no competing interests.

\section{References}

1. Pensel PE, Elissondo N, Gambino G, Gamboa GU, Benoit JP, Elissondo MC. Experimental cystic echinococcosis therapy: in vitro and in vivo combined 5-fluorouracil/albendazole treatment. Vet Parasitol. 2017;245:62-70. doi:10.1016/j.vetpar.2017.08.011

2. Ito A, Nakao M, Lavikainen A, Hoberg E. Cystic echinococcosis: future perspectives of molecular epidemiology. Acta Trop. 2017;165:3-9.

3. Brunetti E, Kern P, Vuitton DA. Expert consensus for the diagnosis and treatment of cystic and alveolar echinococcosis in humans. Acta Trop. 2010;114(1):1-16. doi:10.1016/j.actatropica.2009.11.001

4. Wen H, Vuitton L, Tuxun T, et al. Echinococcosis: advances in the 21st century. Clin Microbiol Rev. 2019;32(2).

5. Wen LM, Lü GD, Zhao J, et al. Molecular cloning and characterization of ribosomal protein RPS9 in echinococcus granulosus. $J$ Parasitol. 2017;103(6):699-707. doi:10.1645/16-164

6. Wang $\mathrm{W}, \mathrm{Li} \mathrm{J}$, Yao J, et al. In vitro and in vivo efficacies of novel carbazole aminoalcohols in the treatment of cystic echinococcosis. J Antimicrob Chemother. 2017;72(11):3122-3130. doi:10.1093/jac/ dkx250
7. Dehkordi AB, Sanei B, Yousefi M, et al. Albendazole and treatment of hydatid cyst: review of the literature. Infect Disord Drug Targets. 2019;19(2):101-104

8. Hemphill A, Stadelmann B, Rufener R, et al. Treatment of echinococcosis: albendazole and mebendazole-what else? Parasite (Paris, France). 2014;21:70.

9. Wong $\mathrm{YK}, \mathrm{Xu} \mathrm{C}, \mathrm{Kalesh} \mathrm{KA}$, et al. Artemisinin as an anticancer drug: recent advances in target profiling and mechanisms of action. Med Res Rev. 2017;37(6):1492-1517.

10. Gopalakrishnan AM, Kumar N. Antimalarial action of artesunate involves DNA damage mediated by reactive oxygen species. Antimicrob Agents Chemother. 2015;59(1):317-325. doi:10.1128/ AAC.03663-14

11. Antoine T, Fisher N, Amewu R, O’Neill PM, Ward SA, Biagini GA. Rapid kill of malaria parasites by artemisinin and semi-synthetic endoperoxides involves ROS-dependent depolarization of the membrane potential. J Antimicrob Chemother. 2014;69(4):1005-1016.

12. Wang J, Huang L, Li J, et al. Artemisinin directly targets malarial mitochondria through its specific mitochondrial activation. PLoS One. 2010;5(3):e9582. doi:10.1371/journal.pone.0009582

13. Ariey F, Witkowski B, Amaratunga C, et al. A molecular marker of artemisinin-resistant plasmodium falciparum malaria. Nature. 2014;505(7481):50-55. doi:10.1038/nature 12876

14. Lichota A, Gwozdzinski K. Anticancer activity of natural compounds from plant and marine environment. Int J Mol Sci. 2018;19(11):3533. doi:10.3390/ijms 19113533

15. Sordi R, Nandra KK, Chiazza F, et al. Artesunate protects against the organ injury and dysfunction induced by severe hemorrhage and resuscitation. Ann Surg. 2017;265(2):408-417. doi:10.1097/ SLA.0000000000001664

16. Zhao D, Zhang J, Xu G, Wang Q. Artesunate protects LPS-induced acute lung injury by inhibiting TLR4 expression and inducing Nrf2 activation. Inflammation. 2017;40(3):798-805. doi:10.1007/s10753017-0524-6

17. Spicher M, Roethlisberger C, Lany $\mathrm{C}$, et al. In vitro and in vivo treatments of echinococcus protoscoleces and metacestodes with artemisinin and artemisinin derivatives. Antimicrob Agents Chemother. 2008;52(9):3447-3450. doi:10.1128/AAC.00553-08

18. Abdel-Baki AA, Almalki E, Mansour L, Al-Quarishy S. In vitro scolicidal effects of salvadora persica root extract against protoscolices of echinococcus granulosus. Korean J Parasitol. 2016;54 (1):61-66. doi:10.3347/kjp.2016.54.1.61

19. Colebrook AL, Jenkins DJ, Jones MK, Tatarczuch L, Lightowlers MW. Effect of cyclosporin A on the survival and ultrastructure of echinococcus granulosus protoscoleces in vitro. Parasitology. 2004;129(4):497-504. doi:10.1017/S00311820040 05773

20. Chen HY, Wu SH. Horseradish peroxidase-encapsulated hollow silica nanospheres for intracellular sensing of reactive oxygen species. Nanoscale Res Lett. 2018;13(1):123.

21. Gopalakrishnan AM, Kumar N, Griffin DE. Opposing roles for two molecular forms of replication protein A in Rad51-Rad54-mediated DNA recombination in plasmodium falciparum. mBio. 2013;4(3): e00252-00213. doi:10.1128/mBio.00252-13

22. Wang H, Li J, Guo B, et al. In vitro culture of echinococcus multilocularis producing protoscoleces and mouse infection with the cultured vesicles. Parasit Vectors. 2016;9(1):411. doi:10.1186/s13071016-1687-y

23. Zhang W-B, Jones MK, Li J, McManus DP. Echinococcus granulosus: pre-culture of protoscoleces in vitro significantly increases development and viability of secondary hydatid cysts in mice. Exp Parasitol. 2005;110(1):88-90. doi:10.1016/j.exppara.2005.02.003

24. Zhu L, He Z, Wu F, et al. Immunization with advanced glycation end products modified low density lipoprotein inhibits atherosclerosis progression in diabetic apoE and LDLR null mice. Cardiovasc Diabetol. 2014;13(1):151. doi:10.1186/s12933-014-0151-6 
25. Antoniou M, Tselentis Y. Studies on Echinococcus granulosus using the scanning electron microscope. I. Preparations of the parasite for infection of the final host. Parasitol Res. 1993;79(7):537-542. doi:10.1007/BF00932236

26. Koppen G, Azqueta A, Pourrut B, Brunborg G, Collins AR, Langie SAS. The next three decades of the comet assay: a report of the 11th International Comet Assay Workshop. Mutagenesis. 2017;32 (3):397-408. doi:10.1093/mutage/gex002
27. Li YF, Wen LM. In vitro and in vivo efficacy of DNA damage repair inhibitor veliparib in combination with artesunate against echinococcus granulosus. Dis Markers. 2020;2020:8259820.

\section{Publish your work in this journal}

Drug Design, Development and Therapy is an international, peerreviewed open-access journal that spans the spectrum of drug design and development through to clinical applications. Clinical outcomes, patient safety, and programs for the development and effective, safe, and sustained use of medicines are a feature of the journal, which has also been accepted for indexing on PubMed Central. The manuscript management system is completely online and includes a very quick and fair peer-review system, which is all easy to use. Visit http://www. dovepress.com/testimonials.php to read real quotes from published authors. 